\title{
The Optimal Suture Placement and Bone Tunnels for TFCC Repair: A Cadaveric Study
}

\author{
Taiichi Matsumoto, $\mathrm{MD}^{1}$ Peter Tang, MD, $\mathrm{MPH}^{2}$ \\ Melvin P. Rosenwasser, $\mathrm{MD}^{4}$ \\ ${ }^{1}$ Department of Orthopaedic Surgery, Kurashiki Central Hospital, \\ Okayama, Japan \\ 2 Department of Orthopaedic Surgery, Allegheny General Hospital, \\ Allegheny Health Network, Pittsburgh, Pennsylvania \\ 3 Department of Orthopaedic Surgery, Kansai Electric Power Hospital, \\ Osaka, Japan \\ ${ }^{4}$ Department of Orthopaedic Surgery, Columbia University Medical \\ Center, New York, New York
}

J Wrist Surg 2018;7:375-381.
Keiji Fujio, $M D^{3} \quad$ Robert J. Strauch, $M^{4}$

Address for correspondence Taiichi Matsumoto, MD, Department of Orthopaedic Surgery, Kurashiki Central Hospital, 1-1-1 Miwa Kurashiki, Okayama 710-8602, Japan

(e-mail: tm10442@kchnet.or.jp).

\begin{abstract}
Keywords

- triangular fibrocartilage complex

- foveal

- transosseous repair

- cadaveric

- optimal suture location

Background Transosseous repair of foveal detachment of the triangular fibrocartilage complex (TFCC) is effective for distal radioulnar joint stabilization. However, studies of the optimal foveal and TFCC suture positions are scant.

Purpose The purpose of this study was to clarify the optimal TFCC suture position and bone tunnels for transosseous foveal repair.

Materials and Methods Seven cadavers were utilized. The TFCC was incised the foveal insertion and sutured at six locations (TFCCs 1-6) using inelastic sutures. Six osseous tunnels were created in the fovea (foveae 1-6). Fovea 2 is located at the center of the circle formed by the ulnar head overlooking the distal end of the ulna (theoretical center of rotation); fovea 5 is located $2 \mathrm{~mm}$ ulnar to fovea 2 . TFCC 5 is at the ulnar apex of the TFCC disc; TFCC 4 is $2 \mathrm{~mm}$ dorsal to TFCC 5. TFCC 1 to 6 sutures were then placed through each of the six osseous tunnels, resulting in 36 combinations, which were individually tested. The forearm was placed in five positions between supination and pronation, and the degree of suture displacement was measured. The position with the least displacement indicated the isometric point of the TFCC and fovea.

Results The mean distance of suture displacement was $2.4 \pm 1.6 \mathrm{~mm}$. Fovea 2, combined with any TFCC location, $(0.7 \pm 0.6 \mathrm{~mm})$ and fovea group 5 , combined with TFCC 4 location $(0.8 \pm 0.8)$ or with TFCC 5 location $(0.9 \pm 0.6)$ had statistically shorter suture displacements than any other fovea groups.

Conclusion For TFCC transosseous repair, osseous tunnel position was more important than TFCC suture location.
\end{abstract}

Triangular fibrocartilage complex (TFCC) injury can lead to distal radioulnar joint (DRUJ) instability and ulnar-sided wrist pain. The deep portion of the TFCC inserts in the fovea of the ulnar head and is critical to DRUJ stability. There are numerous methods to repair the TFCC, but there is no consensus on whether transcapsular suture ${ }^{1-3}$ or transosseous suture $e^{4-9}$ is more effective.

The optimal suture location of the TFCC and fovea should allow full range of movement at the wrist without causing long-term degeneration of the TFCC suture site. Only a few received

December 23, 2016

accepted

May 21, 2018

published online

June 26, 2018
Copyright $\odot 2018$ by Thieme Medical Publishers, Inc., 333 Seventh Avenue, New York, NY 10001, USA. Tel: +1(212) 584-4662.
DOI https://doi.org/ 10.1055/s-0038-1661361. ISSN 2163-3916. 


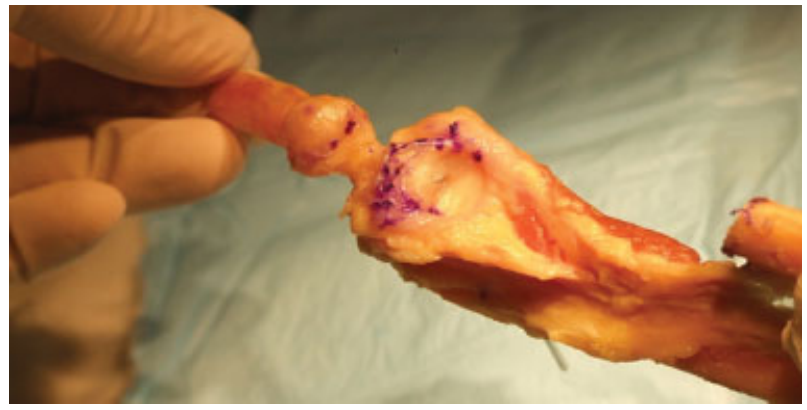

Fig. 1 The ulnar shaft was osteotomized $5 \mathrm{~cm}$ from the distal ulna and inverted. Then the TFCC was incised at the fovea. The dotted line marks the margin of the TFCC annular disc. TFCC, triangular fibrocartilage complex.

studies discussed the optimal site of suture placement in the fovea and TFCC for transosseous suture repair. To clarify the optimal site of suture placement, we used an anatomical model to investigate various suture positions in the TFCC and fovea. The model was based on studies undertaken to clarify the optimal site of bone tunnel placement in anterior cruciate ligament $(\mathrm{ACL})$ reconstruction in the knee. ${ }^{10,11}$ Hoogland and Hillen ${ }^{10}$ measured the length pattern of 12 different positions (three femoral tunnels and four tibial tunnels) from extension to flexion with a plastic-coated steel cord fixated to the tibial tuberosity with a staple and placed through the femoral and tibial tunnels under a tension of $5 \mathrm{~kg}$. The change in the cord's length was measured between extension and flexion to determine the tunnel position at which there are minimal length changes in the steel cord. We adapted this measurement method in our anatomical model. The biomechanics of the ACL and TFCC are very different, but the deep portion of the TFCC connects with the TFCC disc and fovea in the same manner as the ACL connects with the femur and tibia. This study aimed to investigate the locations where there are minimal length changes in the sutures between the
TFCC and the foveal tunnels and the ideal reconstruction route in the event of injury to the foveal ligament connecting the two components.

\section{Materials and Methods}

We used seven fresh-frozen forearms with elbows (four from the right and three from the left) from three male and four female white cadavers with a mean age of 72.9 years (range, 63-83 years). Before the study, all musculature was removed, and the radiocarpal joint was disarticulated. All ligaments and the interosseous membrane of the forearm were preserved except $5 \mathrm{~cm}$ from the distal end of the ulna. The ulnar shaft was osteotomized $5 \mathrm{~cm}$ from the distal end and inverted, and the TFCC was divided from the fovea ( $\mathbf{- F i g . ~ 1 ) . ~ T h e ~ o s t e o t o m i z e d ~}$ ulna was stabilized by a five-hole plate and $3.5-\mathrm{mm}$ cortical screws (Stryker Small Fragment Set; Stryker, Mahwah, NJ). In addition, before cutting the ulna, the plate was predrilled and prepositioned. The elbow was fixed at 90 degrees flexion using a 2.4-mm K-wire inserted from the proximal ulna to the distal humerus, and the ulna was mounted to a customized jig. The setup allowed for supination and pronation of the radius around the ulna (-Fig. 2).

-Fig. 3 illustrates the suture locations in the TFCC and fovea. The TFCC reference marker, TFCC 5 , was placed at the ulnar apex of the TFCC disc, whereas the foveal reference marker, fovea 2, was placed at the center of the circle formed by the ulnar head overlooking the distal end of the ulna from an end-on view (i.e., fovea 2 is the theoretical center of rotation) (-Fig. 4).

The TFCC was sutured at six points (TFCCs 1-6) using inelastic sutures (Stealth Code Red Braid, $0.22 \mathrm{~mm}$; Spider Wire, Spirit Lake, IA) by horizontal mattress technique ( - Figs. 5 and 6). There were two limbs on the suture of each TFCC point; the bite distance of the horizontal mattress was $1.5 \mathrm{~mm}$, and the distance between the respective suture locations was
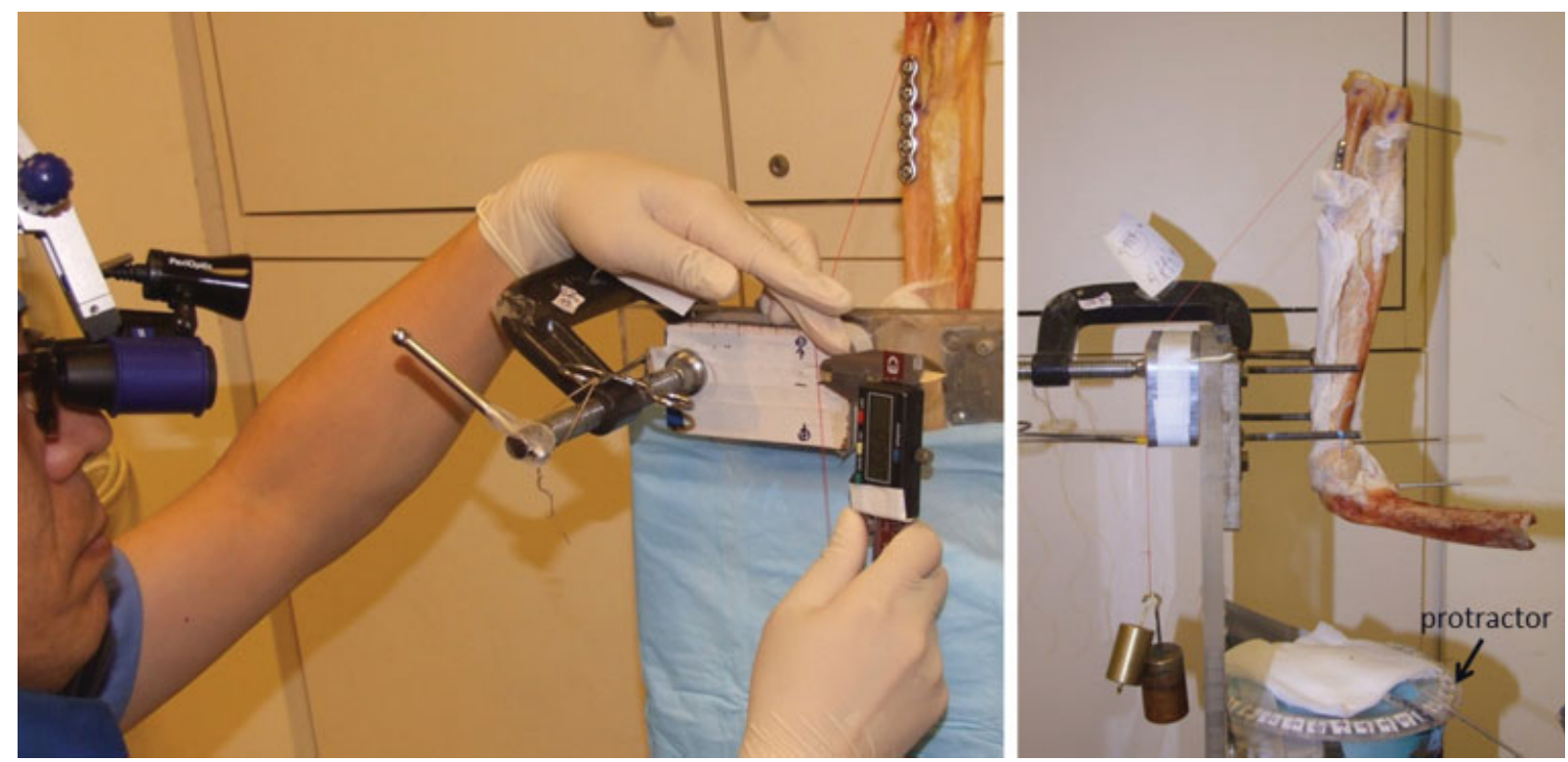

Fig. 2 The ulna was mounted to a customized jig. The suture line moved distally and proximally accompanying with the forearm rotation. The degree of suture displacement was measured using a digital caliper through a loupe at the five predetermined rotation positions. 


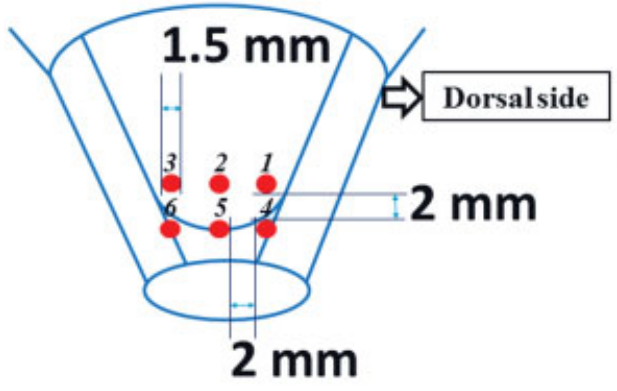

TFCC side lines:

6 different points

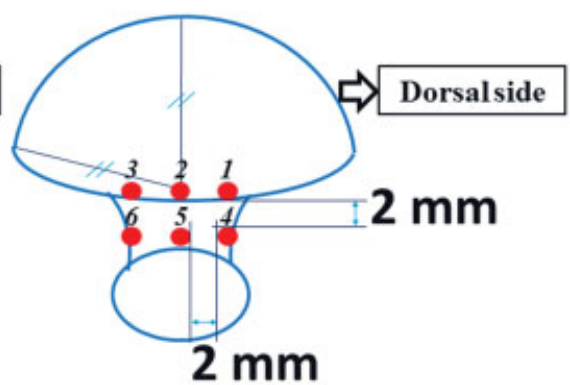

Foveal side lines:

\section{We examined $6 \times 6=36$ combinations.}

Fig. 3 Diagrammatic representation of the suture locations on the TFCC and the fovea. TFCC, triangular fibrocartilage complex.

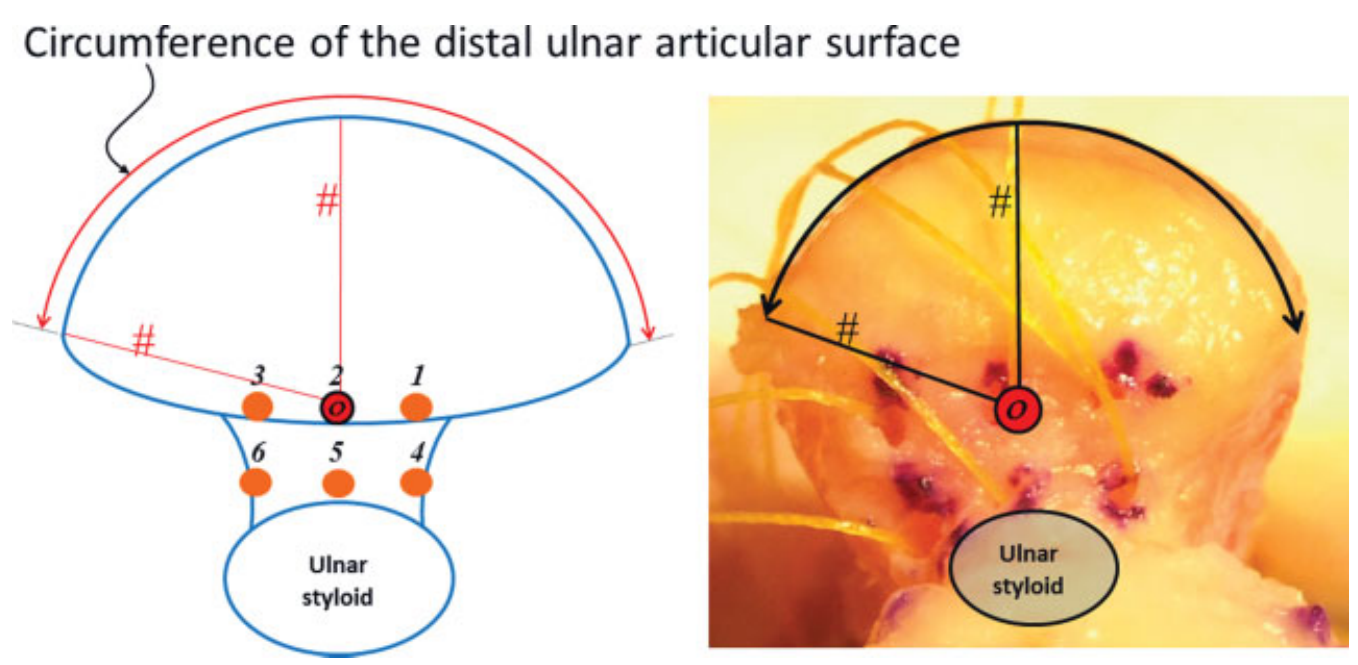

Fig. 4 Ulna from an end-on view. The circumference of the distal ulnar articular surface is identified as a precise circle, with its radius marked with sharp sign (\#). O indicates the position of fovea 2.

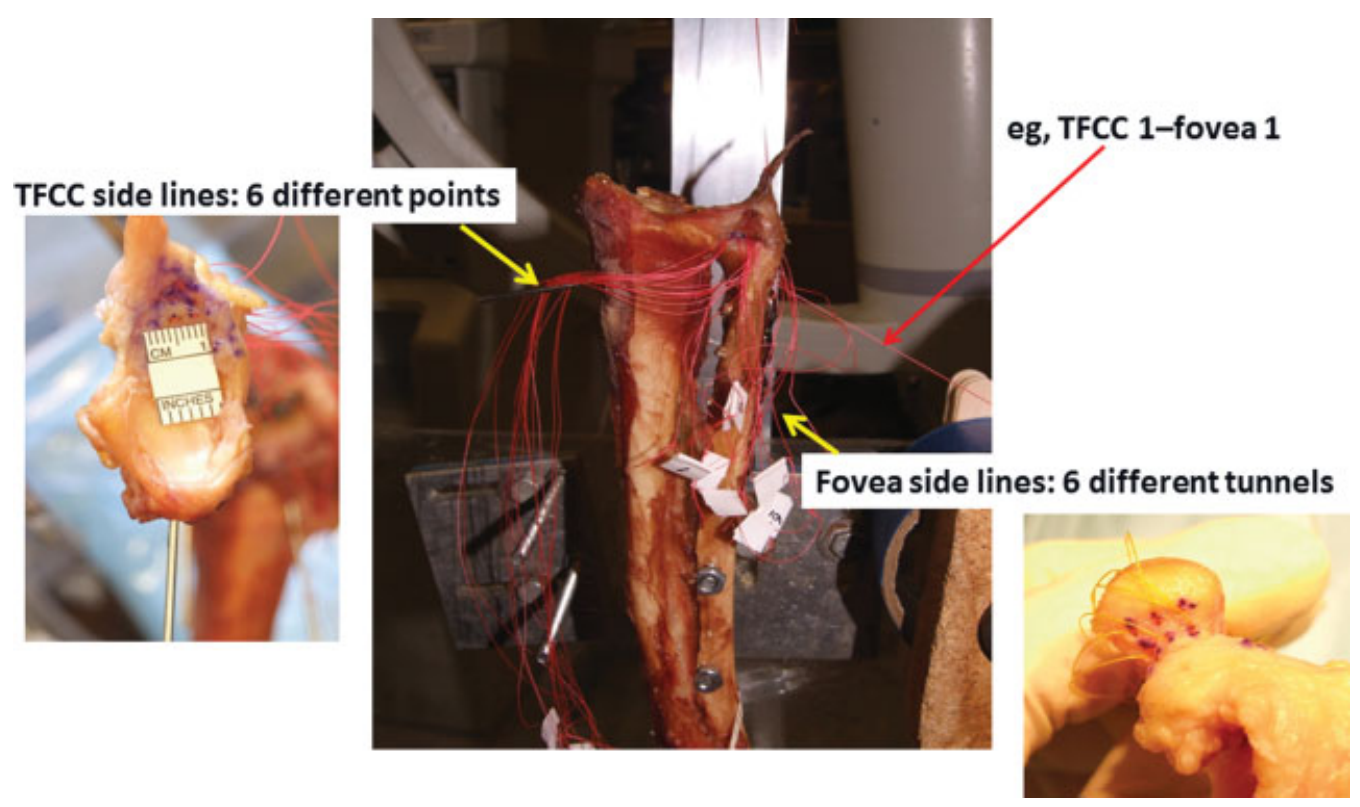

Fig. 5 The six suture locations in the TFCC and six bone tunnels in the fovea. The suture was passed from the TFCC to the foveal bone tunnels. The red arrow points to the suture combination TFCC 1 -fovea 1 . TFCC, triangular fibrocartilage complex. 

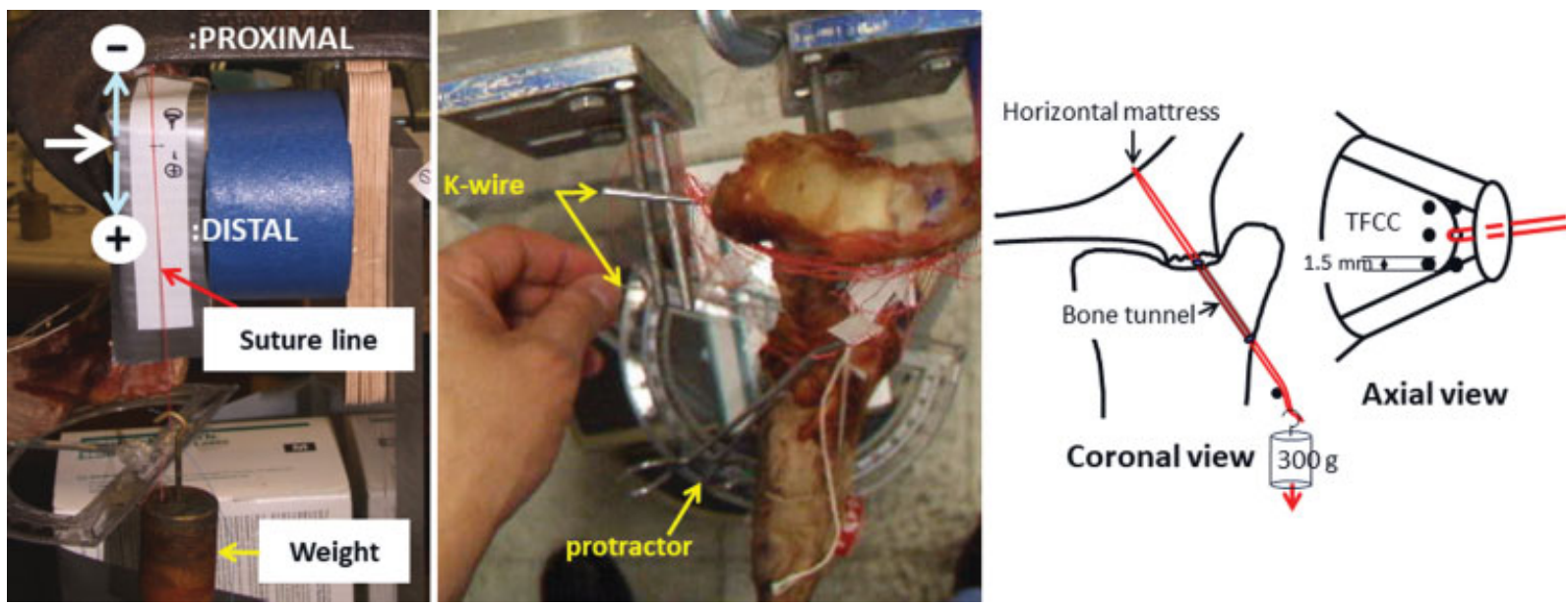

Fig. 6 Displacement of the suture line. The suture line moved distally and proximally (white arrow) as the forearm was manually rotated. Two Kwires inserted in the radius are parallel, and the K-wire points to the protractor. TFCC, triangular fibrocartilage complex.

$2 \mathrm{~mm}$ (-Fig. 3). Six osseous tunnels were created in the fovea (foveae 1-6). A small bony tunnel was created in the fovea with a 19-gauge needle, and the distance between tunnels was $2 \mathrm{~mm}$. Each of the six TFCC sutures was then placed through each of the six bone tunnels, resulting in 36 combinations of TFCC locations and foveal tunnels ( - Figs. 3 and $\mathbf{5}$ ). A weight of $300 \mathrm{~g}$ was applied to each suture (-Fig. 6).

From each of the five positions between maximal supination and maximal pronation (maximal supination, 45 degrees of supination, neutral position, 45 degrees of pronation, and maximal pronation), the degree of suture displacement was measured using a digital caliper (resolution, $0.01 \mathrm{~mm}$; Carrera Precision, Guangdong, China) (-Fig. 6). The radius is manually rotated to these five positions, which are set by pointing a Kwire inserted into the radius of the protractor ( - Figs. 2 and $\mathbf{6}$ ). All measurements were taken at the same time interval after applying the 300-g weight to allow the soft tissue mattress to recover between each loading. The maximum and minimum lengths of the suture line were measured during forearm rotation, and the difference was defined as the suture displacement. An optimal suture location was defined as having the shortest displacement distance.

\section{Statistical Analysis}

Two-way analysis of variance and multiple comparison analysis were performed. The analysis factors were TFCC, fovea, and TFCC $\times$ fovea. The IBM SPSS Statistics 20 software (Statistical Package for Social Sciences, Armonk, NY) was used for the statistical analyses. A $p$-value of $<0.05$ was considered statistically significant.

\section{Results}

The mean suture displacement was $2.4 \pm 1.6 \mathrm{~mm}$ (range, 0-6.2 $\mathrm{mm}$ ) over the entire range of forearm rotation. The mean forearm pronation was $76.0 \pm 8.7$ degrees (range, 20-90 degrees), and all specimens had forearm pronation greater than 65 degrees in all 36 combinations except one specimen with 20 degrees in the TFCC 1 -fovea 1 combination. The mean forearm supination was
$76.5 \pm 7.4$ degrees (range, 55-90 degrees), and all of the values had forearm supination greater than $70^{\circ}$ except two specimens with 55 degrees in TFCC 1 -fovea 1 combination.

The homogeneity of variance held for any combination of all levels in Levine's test for equality of variance $(p=0.086)$. Statistical analysis was performed on the fovea because the interaction between the TFCC and the fovea demonstrated significant difference in the between-subjects effects $(p=0.001)$.

Subeffect tests were performed using the Bonferroni method to test pairwise comparisons among foveae 1 to 6 in each TFCC position. There are significant differences in foveae $1,3,4$, and 6 (all $p<0.005)$, but none in foveae $2(p=1.00)$ and $5(p>0.2)$,

Table 1 Results of the two-way analysis of variance and multiple comparison analysis

\begin{tabular}{|l|l|l|l|}
\hline Fovea & $($ I) TFCC & $(\mathrm{J})$ TFCC & $p$-Value \\
\hline 1 & 1 & 3 & 0.001 \\
\hline & 2 & 4 & 0.007 \\
\hline & & 5 & 0.018 \\
\hline & 3 & 4 & 0.000 \\
\hline & & 5 & 0.000 \\
\hline 3 & 3 & 5 & 0.039 \\
\hline 4 & 1 & 4 & 0.037 \\
\hline & & 5 & 0.001 \\
\hline & 2 & 5 & 0.042 \\
\hline & 3 & 5 & 0.004 \\
\hline 6 & 1 & 3 & 0.000 \\
\hline & 3 & 4 & 0.000 \\
\hline & & 5 & 0.002 \\
\hline & 4 & 6 & 0.013 \\
\hline
\end{tabular}

Abbreviation: TFCC, triangular fibrocartilage complex.

${ }^{a}$ Only values showing significant differences are presented here $(p<0.05)$. 
Table 2 Results of the Kruskal-Wallis' analysis

\begin{tabular}{|c|c|}
\hline Sample 1 -sample 2 & Adjusted $p$-Value ${ }^{a}$ \\
\hline Fovea 2 -fovea 1 & 0.000 \\
\hline Fovea 2-fovea 3 & 0.000 \\
\hline Fovea 2-fovea 4 & 0.000 \\
\hline Fovea 2-fovea 6 & 0.000 \\
\hline Fovea 5-fovea 1 & 0.003 \\
\hline Fovea 5-fovea 3 & 0.001 \\
\hline Fovea 5-fovea 4 & 0.000 \\
\hline Fovea 5-fovea 6 & 0.000 \\
\hline Fovea 2-fovea 5 & 0.069 \\
\hline
\end{tabular}

a Only values showing a significant difference $(p<0.05)$ are presented; fovea 2 -fovea 5 values are presented here.

which means that there is no significant difference in suture displacement among the TFCC groups belonging to foveae 2 and 5 , respectively. The mean suture displacement for fovea 2 was $0.7 \pm 0.6 \mathrm{~mm}$ (range, $0-2.4 \mathrm{~mm}$ ), and the mean suture displacement for fovea 5 was $1.5 \pm 0.8 \mathrm{~mm}$ (range, $0.1-3.0 \mathrm{~mm}$ ). The significant differences among all pairs of means in this study are shown in - Table $\mathbf{1}(p<0.05)$.

In an additional statistical analysis, we compared each fovea group, based on the data points of TFCC 1 to 6 , using the Kruskal-Wallis' analysis for multiple comparisons (-Table 2). The fovea 2 group had significantly shorter suture displacement than the other fovea groups, except for the fovea 5 group. The fovea 5 group also had significantly shorter suture displacement than the other fovea groups, except for the fovea 2 group. The $p$-value for the fovea 2 group versus the fovea 5 group was 0.069 , which was very close to a level of significance of 0.05 . Therefore, the fovea 2 group had the shortest values and was followed by the fovea 5 group ( - Figs. 7 and $\mathbf{8}$ ). - Fig. 8 shows that the dotted line of fovea 2 has the minimum displacement regardless of TFCC locations (range, $0.4-0.9 \mathrm{~mm}$ ). We believe that clinically, a displacement of $0.9 \mathrm{~mm}$ is acceptable because it represents very little movement and may not cutout TFCC substance even with repeated wrist movement. Accordingly, $0.9 \mathrm{~mm}$ was set as the upper limit value of the acceptable range; the data for fovea 2 , fovea 5-TFCC 4 , and fovea 5-TFCC 5 conform with this range.

\section{Discussion}

TFCC injury can result in ulnar-sided wrist pain and loss of wrist function. ${ }^{7}$ In particular, the deep portion of the TFCC acts as the main stabilizer of the DRUJ, and any disruption to the foveal insertion can result in instability. ${ }^{12}$ The use of the transosseous suture technique for repairing lost TFCC foveal insertion integrity has been widely reported to be very effective in restoring DRUJ stability. ${ }^{4-9,13,14}$

To repair a TFCC foveal insertion, Nakamura et al ${ }^{8}$ passed a suture through the TFCC and a bone tunnel that was placed at the foveal isometric point. Atzei et $\mathrm{al}^{13,14}$ sutured the TFCC by inserting a suture anchor under a fluoroscope. Iwasaki and Minami ${ }^{9}$ reported successful TFCC suture repair with one small osseous tunnel in the fovea using a $2.9-\mathrm{mm}$ cannulated drill.

However, to the best of our knowledge, there were no reports on whether the optimal suture location was in the TFCC substance or the fovea. The purpose of this study was to clarify the best location for transosseous repair.

The radius rotates around the ulna, and the theoretical rotation center is the center of a circle, if the distal ulnar head is considered a circle. This theoretical rotation center has been regarded as the isometric point. Therefore, the TFCC has been sutured to this theoretical rotation center in the past. For the purposes of our study, fovea 2 was chosen as the

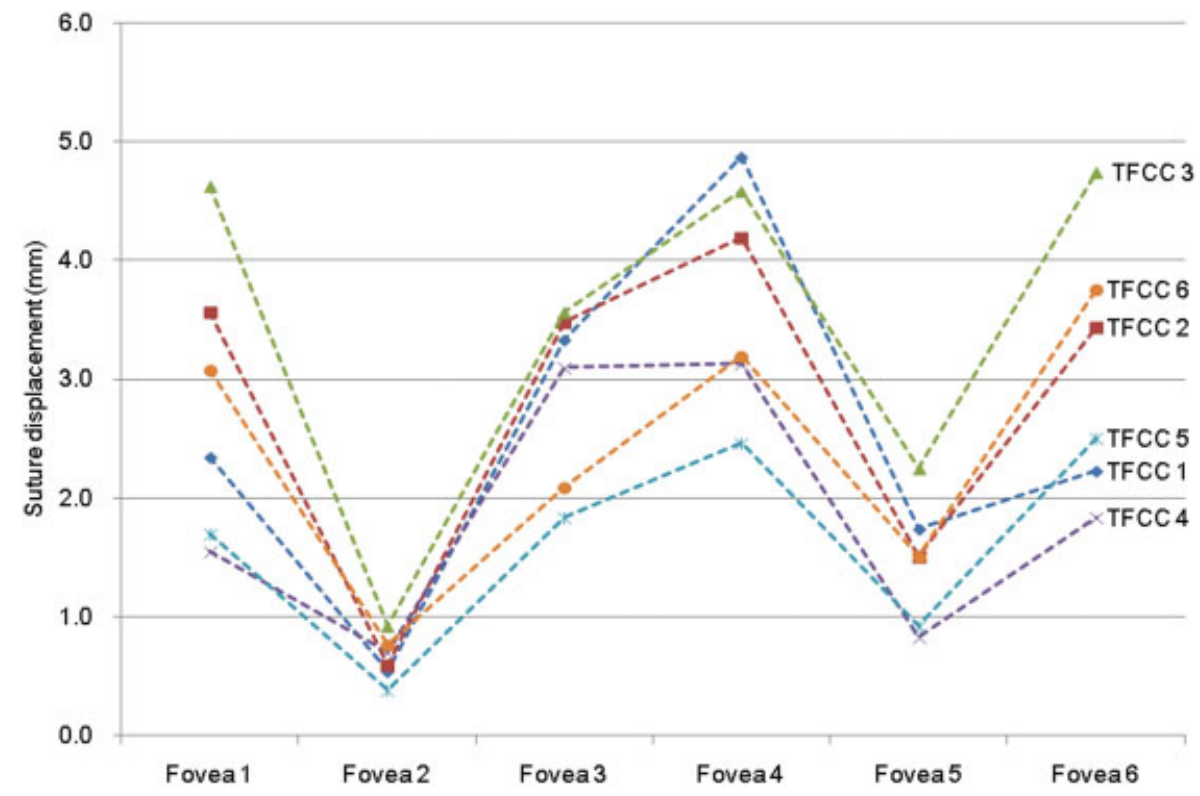

Fig. 7 Suture displacement distance of the different fovea-TFCC combinations. Foveae 2 and 5 have substantially smaller degrees of movement. TFCC, triangular fibrocartilage complex. 


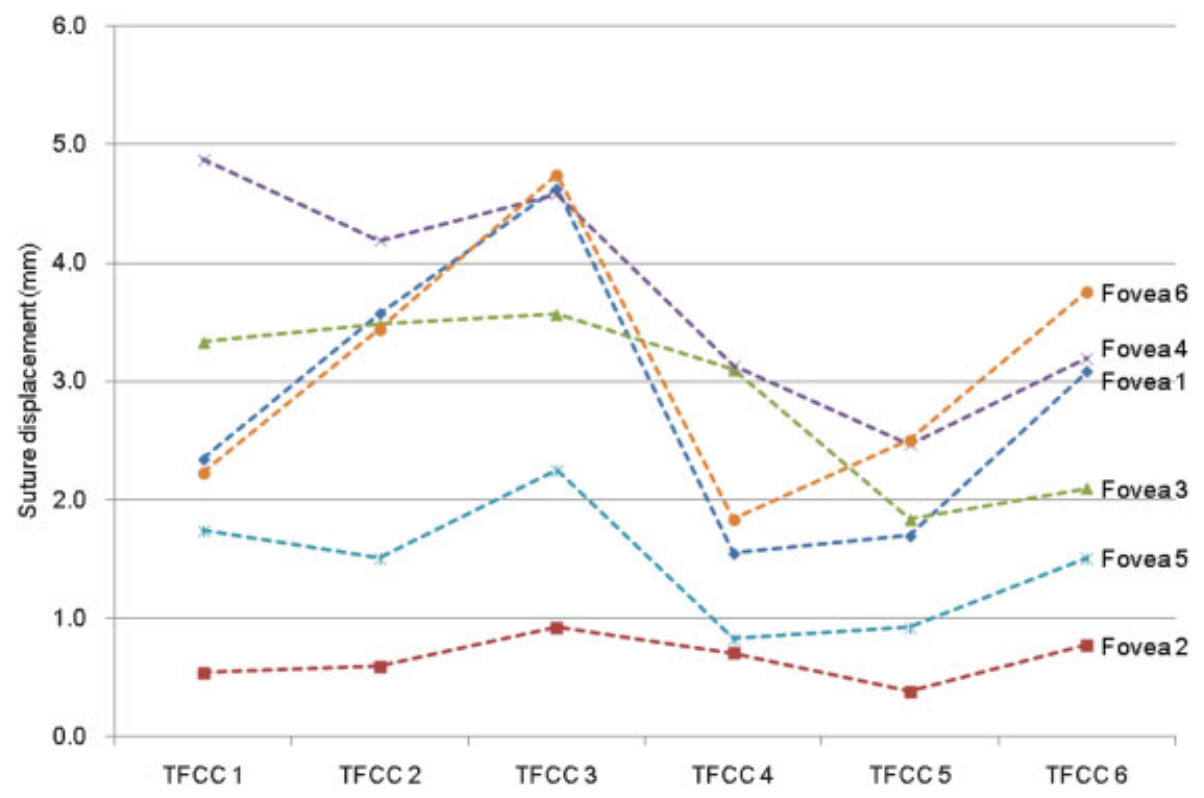

Fig. 8 Suture displacement distance of the different fovea-TFCC combinations shown in - Fig. 7, with the TFCCs in the $x$-axis. TFCC, triangular fibrocartilage complex.

theoretical center of rotation and reference marker, which was similar to the process of identifying the isometric point in ACL reconstruction in the knee. ${ }^{10,11}$ We examined the optimal suture location for both the fovea and the TFCC for transosseous repair in a similar method to that used to examine the optimal positions for femoral and tibial osseous tunnels for ACL reconstruction.

This study has several limitations. First, the cadavers were of old age, resulting in less elastic TFCC tissue, which may not be representative of clinical reality. Second, the ulnocarpal ligaments were transected in the cadavers because the specimens were disarticulated at the wrist. However, this study focused on a transosseous TFCC repair model to establish optimal suture fixation; therefore, only the deep portion of the TFCC and its relationship to the fovea were evaluated. We did not evaluate the superficial portion of the TFCC, the extensor carpi ulnaris subsheath, the ulnolunate ligament, the ulnotriquetral ligament, or the interosseous membrane of the forearm in this study. It is important to ensure the integrity of the TFCC in each specimen. If there was a tear, secondary stabilizing structures could be stretched, causing a different pattern of motion during supination and pronation, but there was no apparent tear in any of the specimens.

Despite the aforementioned limitations, we believe we have useful results that can be translated to clinical use. According to our data, the optimal bone tunnels on the fovea for transosseous TFCC repair include foveae 2 and 5. Interestingly, if any of the TFCC locations is repaired at the location of fovea 2 , there is minimal suture displacement. If fovea 5 is used, the optimal TFCC location is 4 or 5. Practically, this means that the surgeon should aim for fovea 2 and TFCC 4 or 5 locations to attach the TFCC successfully to the foveal isometric point. The challenge would be to find this center of the distal ulnar circle intraoperatively, when exposure is not as extensive as in cadaveric laboratory setting.

\section{Note}

This study was done at the Anne Youle Stein Center for Orthopedic Education and Research in Columbia University Medical Center, New York, NY.

\section{Conflict of Interest}

None.

\section{Acknowledgment}

The authors thank Mr. Yoshiharu Yamamoto (Consultation Division, Clinical Research Support Center, Kurashiki Central Hospital) for advice and assistance in handling statistical data.

\section{References}

1 Haugstvedt JR, Husby T. Results of repair of peripheral tears in the triangular fibrocartilage complex using an arthroscopic suture technique. Scand J Plast Reconstr Surg Hand Surg 1999;33(04):439-447

2 Millants P, De Smet L, Van Ransbeeck H. Outcome study of arthroscopic suturing of ulnar avulsions of the triangular fibrocartilage complex of the wrist. Chir Main 2002;21(05):298-300

3 Yao J. All-arthroscopic triangular fibrocartilage complex repair: safety and biomechanical comparison with a traditional outsidein technique in cadavers. J Hand Surg Am 2009;34(04):671-676

4 Moritomo $\mathrm{H}$. Advantages of open repair of a foveal tear of the triangular fibrocartilage complex via a palmar surgical approach. Tech Hand Up Extrem Surg 2009;13(04):176-181

5 Tang C, Fung B, Chan R, Fok M. The beauty of stability: distal radioulnar joint stability in arthroscopic triangular fibrocartilage complex repair. Hand Surg 2013;18(01):21-26 
6 Shinohara T, Tatebe M, Okui N, Yamamoto M, Kurimoto S, Hirata $\mathrm{H}$. Arthroscopically assisted repair of triangular fibrocartilage complex foveal tears. J Hand Surg Am 2013;38(02): 271-277

7 Hermansdorfer JD, Kleinman WB. Management of chronic peripheral tears of the triangular fibrocartilage complex. J Hand Surg Am 1991;16(02):340-346

8 Nakamura T, Sato K, Okazaki M, Toyama Y, Ikegami H. Repair of foveal detachment of the triangular fibrocartilage complex: open and arthroscopic transosseous techniques. Hand Clin 2011;27 (03):281-290

9 Iwasaki N, Minami A. Arthroscopically assisted reattachment of avulsed triangular fibrocartilage complex to the fovea of the ulnar head. J Hand Surg Am 2009;34(07):1323-1326

10 Hoogland T, Hillen B. Intra-articular reconstruction of the anterior cruciate ligament. An experimental study of length changes in different ligament reconstructions. Clin Orthop Relat Res 1984; (185):197-202

11 Penner DA, Daniel DM, Wood P, Mishra D. An in vitro study of anterior cruciate ligament graft placement and isometry. Am J Sports Med 1988;16(03):238-243

12 Haugstvedt JR, Berger RA, Nakamura T, Neale P, Berglund L, An KN. Relative contributions of the ulnar attachments of the triangular fibrocartilage complex to the dynamic stability of the distal radioulnar joint. J Hand Surg Am 2006;31(03):445-451

13 Atzei A. New trends in arthroscopic management of type 1-B TFCC injuries with DRUJ instability. J Hand Surg Eur Vol 2009;34(05): 582-591

14 Atzei A, Rizzo A, Luchetti R, Fairplay T. Arthroscopic foveal repair of triangular fibrocartilage complex peripheral lesion with distal radioulnar joint instability. Tech Hand Up Extrem Surg 2008;12 (04):226-235 